\title{
A Delay Time Overlapping MAC Protocol for Underwater Wireless Sensor Networks
}

\author{
Bok-Rae Jang and Seung-Hyun Oh* \\ Department of Computer Science, Dongguk Univ., S. Korea \\ \{pse5802,shoh\}@dongguk.ac.kr
}

\begin{abstract}
Underwater wireless sensor networks are widely used in environmental monitoring and a variety of applications. However, underwater wireless sensor networks should be handled carefully because they have inherent disadvantages, such as long propagation delay in the acoustic signal and low-bandwidth. If using existing protocols for a terrestrial wireless network in an underwater network, performance decreases significantly, so there is a need for a new protocol. This study aims to improve performance that is reduced by a long wait time after a request to send/clear to send (RTS/CTS) transmission in the MAC protocol handshake. By utilizing the wait time after transmitting a packet, sending packets that can be transferred to a neighbor Node Can provide better performance. Experimental results show that end-to-end delay and throughput performance are significantly improved, compared to the existing well-known protocol.
\end{abstract}

Keywords: Time Reuse, Underwater Acoustic Networks, Handshaking, MAC protocol

\section{Introduction}

Wireless sensor networks are being developed for the Internet of Things (IoT), and recently, research has attracted much attention as it has advanced. Compared to wireless sensor networks that send and receive packets through an electromagnetic wave signal, an underwater wireless sensor network uses sound waves. Because an electromagnetic wave is severely attenuated in water, long-distance transmission is difficult. The use of underwater wireless sensor networks in a variety of fields, like underwater environmental monitoring, natural resource development, and military applications, has increased. However, communications using sound waves has various problems.

Typically, there is very low bandwidth and a slow propagation delay rate. Propagation delay issues bring problems that were not considered a weak point for ground-based electromagnetic wave-transmission environments. This is why it becomes difficult to use a conventional wireless sensor network protocol. So, underwater wireless sensor networks need a new protocol that takes the limitations into account. A medium access control (MAC) protocol that uses a handshake mechanism can be used to solve the hidden terminal problem in the existing wireless network by using a control message. In other words, the control message can be used to solve the collision problem through reservation of wireless media. The problem of the exposed terminal, however, cannot be solved only with a control message.

In this research, transmission time due to a long propagation delay will be reused when transferring a packet underwater. In underwater communications, transfers

* Corresponding Author 
between packets are very slow due to the long propagation delay. High throughput can be obtained only if transmission time is reused, and as a solution, each node will collect data, storing them in its own table. Once the data are collected, the node will send a packet if it is located near enough to a neighboring node. Additionally, a measure to solve the exposed terminal problem with MAC protocols will be introduced. Finally, a new underwater-based MAC protocol that can enhance throughput will be suggested. The proposed MAC protocol aims to improve the throughput performance through the overlapping the time period of waiting times of almost all phase of data frame transmission with other side neighbor node transmission. When one node exchange data with node, it must wait long time due to slow acoustic sound wave signal in the underwater environment, so the proposed MAC protocol can get improved performance.

In the chapter 2, we will review related preceding researches, and in the chapter 3 , we will explains our proposed MAC protocol. Chapter 4 and 5 will show the simulation experiment results of our protocol and conclusions. In the conclusion, we will discuss the future research topics.

\section{Related Works}

A considerable amount of research into MAC protocols in wireless network environments has been done, and even now, MAC protocols that enhance throughput are suggested with various methods. Since most research has focused on above-ground, electromagnetic frequency wireless networks, the rate of underwater wireless network development is much more staggered. The main reason is that the transfer method for packets is different in underwater environments compared to above-ground environments. Above ground, radio frequency (RF) is used to transfer data, but an RF signal is attenuated very rapidly and cannot be used under water because more factors must be considered. In fact, if a packet is transferred underwater, throughput becomes substantially low, compared to that of an aboveground wireless network, due to low propagation delay, a high multipath, the fading effect, and limited bandwidth [1-2].

To solve such problems, Guo et al [3-4] suggested a protocol that is strong despite a long propagation delay. This protocol transformed the existing handshaking mechanism. In other words, after sending an RTS, the CTS is not sent immediately as a response; instead, the suggested method sends the CTS after a certain waiting period. Collisions between frames can be decreased by adding the wait time, but the problem is that the exchange time between RTS and CTS increases as a result, making the whole process less efficient.

$\mathrm{Ng}$ et al [5] suggested Media Access Carrier Avoid-Underwater (MACA-U), a protocol that emerged from transforming the existing MACA protocol. MACA-U is a protocol that adds a state, decreases collisions, and limits transmissions by setting a timer when the corresponding state alters when an exchange between frames occurs in the existing MACA protocol. This protocol adds a state and takes control between frames, which decreases collisions as a result. However, the problem is that every time alteration of the state takes place, time is wasted in setting the timer.

Yun et al. [6] suggested an idea to solve the energy waste that occurs when the existing MAC protocol recognizes a neighbor node. In the research, beacon signals are sent to neighbor nodes, allowing each node to record the existence of surrounding nodes. Fields in the beacon signals include information about the transmission time schedule, and nodes that receive beacon signals manage each transmission time. If beacon signals fail to be received due to a flaw in the channel or a problem in the network, it rearranges the schedule, by sending a joining message. Then, it sends a reservation packet (RP), reserves a transfer with 
information that is in the RP, and then begins the transfer. However, the problem in such research is that it cannot detect a collision when a propagation delay occurs.

Additionally, research comparing performances of protocols that are used under water [7] was conducted, and various protocols that are formed by transforming existing MAC protocols [8-10] were suggested.

In this research, when a long propagation delay exists, a protocol that transmits the data when the neighbor node is adjacent enough was suggested. Also, a solution for the exposed terminal problem (a problem in the existing MAC protocol) is mentioned. The ultimate goal of the protocols suggested is to enhance throughput under water. As a reference, we expanded a conference paper [12] that offered a basic idea for this MAC protocol research.

\section{I-TBPP Mac Protocol}

The protocol that this research suggests is called the Improved Table Base Prediction Protocol (I-TBPP). In other words, it predicts propagation that occurs while transferring data by using table information, and reuses the transmission time that occurs due to long propagation delay.

The frame structure of I-TBPP comprises an RTS frame, a CTS frame, the data frame, and finally, acknowledge (ACK) frame, which checks whether the transfer process was successful. The frame structure of I-TBPP is shown in figure 1.

The mechanism of the I-TBPP is mainly two steps. The first works like the normal MACA wireless MAC protocol [13], where each node inserts the information necessary for its own table. In the second step, based on data that each Node Collected, the Node Decides whether the transmission time due to long propagation delay can be reused. We try to found more time period that can reuse to transmit other data packet between neighbor nodes, then we finally numbering that as a three cases in the figure 3 .

Fist case in the figure 3 is situation between Node A and Node B. When Node B transmit RTS signal to other side Node $\mathrm{C}$, it should wait a CTS signal before transmit data packet. But due to very long propagation delay, Node B waits long time period. At this time, Node A has a data to transmit to Node B, Node A send RTS signal to Node B. When Node B received a RTS signal, it calculate time period to finish communication with Node A based on the distance to Node A. If Node A is enough close to Node B, Node B will decide that data transmission with A during time period for CTS signal waiting. Case 2 is a similar scenario between same nodes, but it is different from Node B wait a data packet transmission from Node C. Node A can reuse time period before data frame arrival, so it can transmit to Node B.

Final case is happen between Node C and Node D. When Node C received a RTS signal from neighbor node, it will respond with CTS signal if the node can accept data frame, after short time period that situation, Node $\mathrm{C}$ receive another RTS signal from other node, Node $\mathrm{D}$. Node $\mathrm{C}$ try to decide it can finish data exchange from Node D before arrival of data frame from Node B. If Node C get answer yes, it send CTS signal to Node D then Node D can communicate with Node C. 
RTS Frame

\begin{tabular}{|c|c|c|c|c|c|c|}
\hline $\begin{array}{l}\text { Frame } \\
\text { Control }\end{array}$ & Start Time & $\begin{array}{c}\text { Departure } \\
\text { Time }\end{array}$ & \multicolumn{2}{|c|}{$\begin{array}{c}\text { Source } \\
\text { Address }\end{array}$} & $\begin{array}{c}\text { Dest } \\
\text { Address } \\
\end{array}$ & CRC \\
\hline \multicolumn{7}{|c|}{ CTS Frame } \\
\hline $\begin{array}{c}\text { Frame } \\
\text { Control }\end{array}$ & Start Time & $\begin{array}{c}\text { Departure } \\
\text { Time }\end{array}$ & \multicolumn{2}{|c|}{$\begin{array}{c}\text { Source } \\
\text { Address }\end{array}$} & $\begin{array}{c}\text { Dest } \\
\text { Address }\end{array}$ & CRC \\
\hline \multicolumn{7}{|c|}{ DATA Frame } \\
\hline $\begin{array}{c}\text { Frame } \\
\text { Control }\end{array}$ & $\begin{array}{c}\text { Source } \\
\text { Address }\end{array}$ & \multicolumn{2}{|c|}{$\begin{array}{c}\text { Dest } \\
\text { Address }\end{array}$} & $\begin{array}{c}\text { MSDU } \\
\text { ID }\end{array}$ & $\begin{array}{l}\text { Frame } \\
\text { Body }\end{array}$ & CRC \\
\hline \multicolumn{5}{|c|}{ ACK Frame } & & \\
\hline $\begin{array}{c}\text { Frame } \\
\text { Control }\end{array}$ & $\begin{array}{c}\text { MSDU } \\
\text { ID }\end{array}$ & $\mathrm{DA}$ & FCS & & & \\
\hline
\end{tabular}

Figure 1. I-TBPP Frame Structure

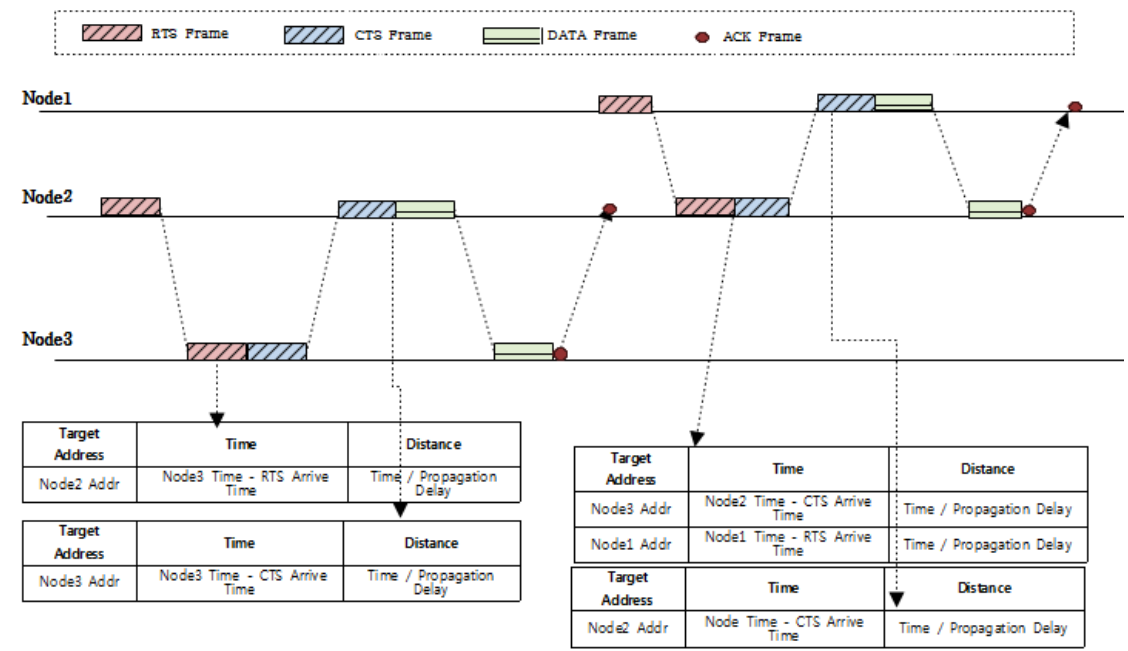

Figure 2. First Step of the I-TBPP

In the first step, each Node collects information, such as target Node Address, frame transmission time, and distance, by exchanging RTS and CTS. Figure 2 shows the RTS and CTS exchange and insertion of table information [12]. The suggested protocol uses this information to reuse the time that occurs due to long propagation delay. The second step is reusing transmission time due to long propagation delay. The total reuse happens in three sections, and information about each section is shown in Figure. 3

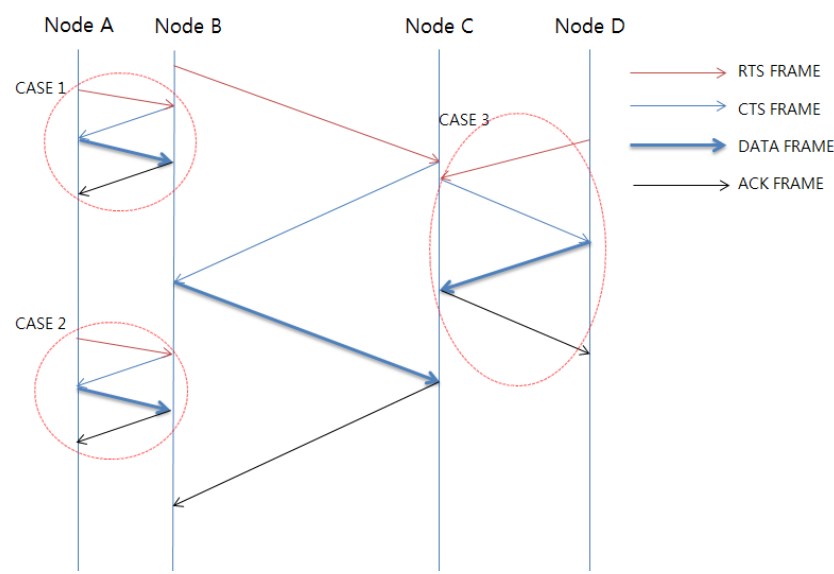

Figure 3. Possible Three Situations in the Transmission Phase 
Three cases are shown in figure 3, each case used to transmit another packet. These cases are used to reuse transmission time that could be list-up as below. Case 1 is receiving RTS from another node while waiting for CTS after sending RTS. Case 2 is receiving RTS from another node while waiting for a data frame, after sending CTS. Finally case 3 is receiving RTS from another node while waiting for ACK, after sending a data frame.

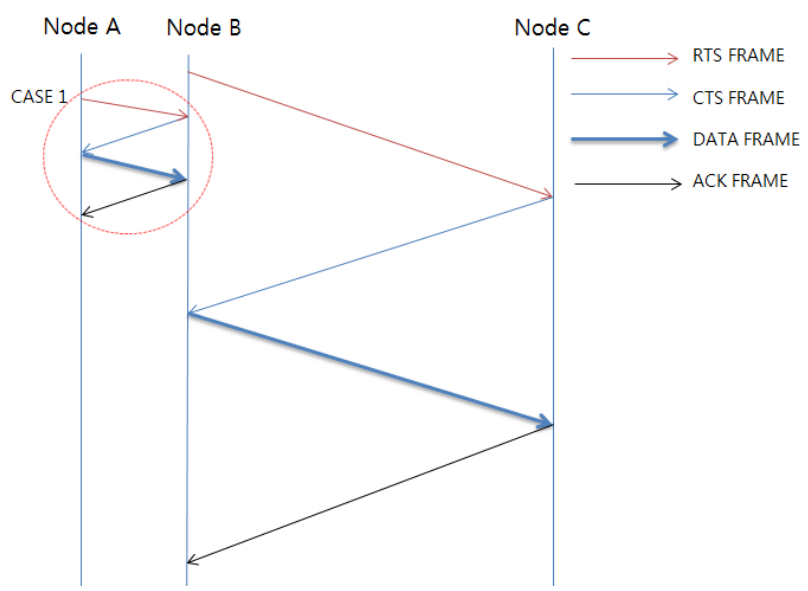

Figure 4. First Case of Overlapping in the Transmission Time

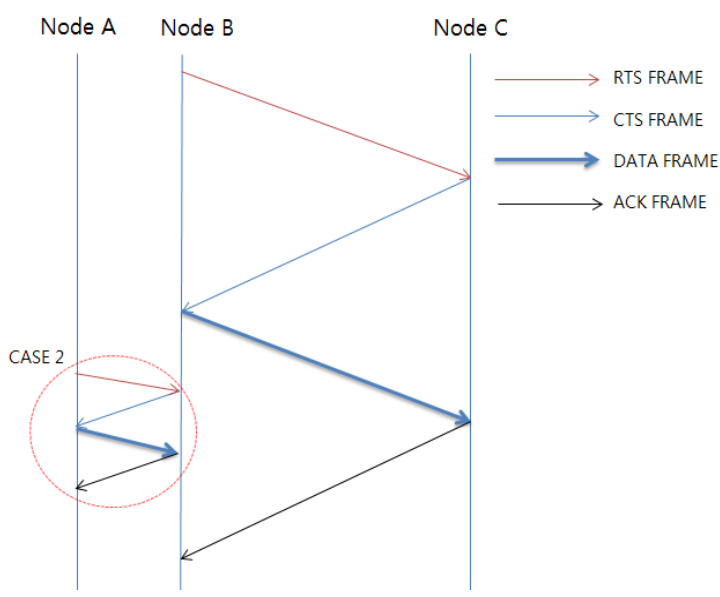

Figure 5. Second Case of Overlapping Scenario

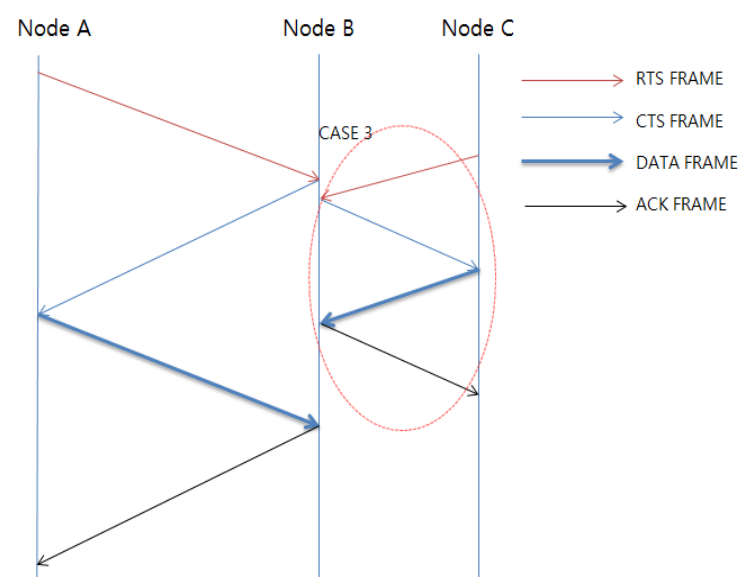

Figure 6. Third Case of Scenario that Node B wait Data Frame 
Case 1 in the figure 4 illustrates Node B transmitting RTS to Node C, and Node B waiting for CTS from Node $\mathrm{C}$, which is the response to RTS. During this process, if Node A transmits RTS to Node B, formula (1) decides whether it is possible to transmit [12].

$$
\mathrm{T}_{\text {prop }}+\text { Distance } \mathrm{A}<\left(\mathrm{T}_{\mathrm{rts}}+\text { Distance } \mathrm{B}\right)-\mathrm{R}_{\mathrm{rts}}
$$

$\mathrm{T}_{\text {prop }}$ from equation (1) is the propagation delay value using the data size of the field value in the RTS frame that was transmitted from Node A to Node B. Distance A is the distance between Node A and Node B, and Distance B is the distance between Node $\mathrm{C}$ and Node $\mathrm{B}$. $\mathrm{T}_{\mathrm{rts}}$ is the time when Node B transmitted RTS to Node $\mathrm{C}$, and $\mathrm{R}_{\mathrm{rts}}$ is the time when Node $\mathrm{B}$ transmitted RTS from Node A.

If " $T_{\text {prop }}+$ Distance A" is smaller than "( $T_{\text {rts }}+$ Distance B $)-R_{r t s}$ " as in (1), Node B transmits CTS to Node A for communicating the data. Afterwards, Node B receives CTS from Node $C$. If the above condition is not satisfied, RTS from Node A is disregarded, and Node B waits for CTS from Node C. Once it receives the CTS frame, data is communicated.

In the second case of figure 5, Node B transmits RTS to Node C. Then, Node C transmits CTS to Node B. Subsequently, Node B transmits the data to Node C. Node B then waits for ACK from Node C. When RTS is transmitted from Node A to Node $\mathrm{B}$, formula (2) will decide whether the transmission is possible [12].

$$
\mathrm{T}_{\text {prop }}+\text { Distance } \mathrm{A}<\left(\mathrm{T}_{\text {data }}+\text { Distance } \mathrm{C}\right)-\mathrm{R}_{\mathrm{rts}}
$$

$\mathrm{T}_{\text {prop }}$ is the propagation delay value using the data size of the field value in the RTS frame that was transmitted from Node A to Node B. Distance A is the distance between Node A and Node B, and Distance B is the distance between Node C and Node B. $T_{\text {data }}$ is the time when Node B transmitted the data frame to Node C, and $\mathrm{R}_{\mathrm{rts}}$ is the time when Node B transmitted RTS to Node A.

If " $T_{\text {prop }}+$ Distance A" is smaller than "( $T_{\text {data }}+$ Distance $\left.C\right)-R_{r t s}$ " as in (2), Node B transmits CTS to Node A to communicate the data. If the above condition is not satisfied, RTS from Node A is disregarded, and Node B waits for ACK from Node C.

In the last case 3 at figure 6, Node A transmits RTS to Node B. Then, Node B transmits CTS to Node A. Subsequently, Node B waits for the data frame from Node A. When RTS is transmitted from Node C to Node B, formula (3) will decide whether the transmission is possible [12].

$$
\mathrm{T}_{\text {prop } 1}+\mathrm{T}_{\text {delay } 1}<\left(\mathrm{CTS}_{\text {prop }}+\mathrm{T}_{\text {prop }}+\mathrm{T}_{\text {delay }}\right)-\mathrm{R}_{\mathrm{rts}}
$$

$\mathrm{T}_{\text {prop } 1}$ is the propagation delay value using the data size of the field value in the RTS frame that was transmitted from Node $C$ to Node $B$, and $T_{\text {delay } 1}$ is its transfer delay value. $\mathrm{CTS}_{\text {prop }}$ is the propagation delay value of the CTS frame transmitted from Node $B$ to Node $A$. $T_{\text {prop }}$ is the propagation delay value using the data size of the field value in the RTS frame that was transmitted from Node A to Node B, and $\mathrm{T}_{\text {delay }}$ is its transfer delay value. $\mathrm{R}_{\mathrm{rts}}$ is the time when Node $\mathrm{B}$ received RTS from Node C.

If " $\mathrm{T}_{\text {prop1 }}+\mathrm{T}_{\text {delay1 }}$ " is smaller than " $\left(\mathrm{CTS}_{\text {prop }}+\mathrm{T}_{\text {prop }}+\mathrm{T}_{\text {delay }}\right)-\mathrm{R}_{\mathrm{rts}}$ " as in (3), Node $\mathrm{B}$ transmits CTS to Node C, communicating the data. If the above condition is not met, RTS from Node C is disregarded, and Node B waits for the data frame from Node A. To sum up, RTS in the middle of the section is disregarded in the three situations when the given formulas are not satisfied. Thus, the node waits for the frame from the previous step. 
The hidden terminal problem can be solved naturally by using RTS and CTS. Reserving the wireless link can ignore different frames when received. However, exposed terminal problems cannot be solved solely through RTS and CTS. The solution for exposed terminal problems in this report is illustrated in figure 7.

Fundamentally, the handshake is transmitted through the broadcast method. For this reason, the nodes within range can listen to the frames of nearby nodes. In the figure 4, Node A's communications range includes Node B, and Node B's communications range includes nodes $\mathrm{C}$ and D. If Node B transmits RTS to Node A, Node $\mathrm{C}$ can also pick it up. Since it is within the broadcasting communications boundary, RTS sent from Node B to Node A can be received by Node C. At this moment, Node $\mathrm{C}$ recognizes that the received RTS was sent to a node other than itself. Node $\mathrm{C}$ sets a timer according to the response standby time for CTS from the received RTS frame field. During this period, if Node $\mathrm{C}$ has data to transmit, and if the CTS frame cannot receive it, Node $\mathrm{C}$ recognizes this as an exposed terminal, and transmits RTS to Node D for communication.

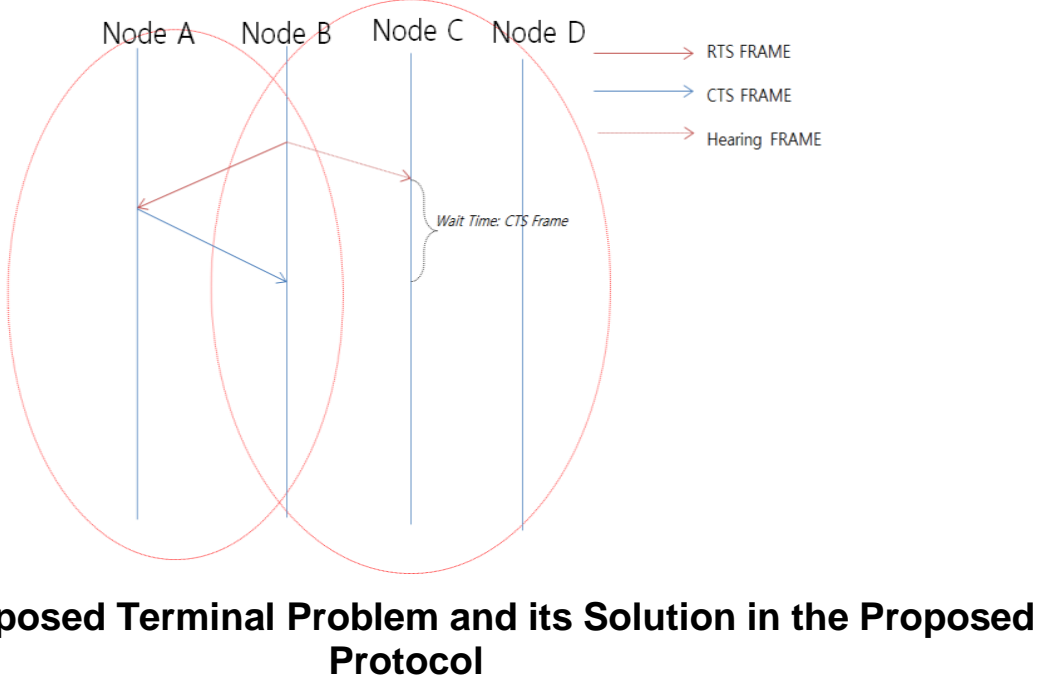

\section{Simulation Results}

For the performance simulation of the proposed protocol, this research used the QualNet network simulation tool. Performance testing was done with MACA, MACAW [11], I-TBPP, and I-TBPP with ACK excluded. For simulation, we deploy 50 nodes with 30 minutes simulation run time, underwater modem's data rate was $4.8 \mathrm{kbps}$. In the simulation, we used an underwater propagation model that gives us a unique signal attenuation model for underwater with acoustic sound wave signal. We simulate scenario 10 times with different seed number, then get an average results.

To evaluate performance, we compare each protocol's performance metrics like end-to-end delay and throughput. Simulation results on end-to-end delay are shown in figure 8. In the overall simulation results, I-TBPP and I-TBPP without ACK had the longest delay. But when offered load in the $\mathrm{x}$-axis increased, their delay times are slowly curved, it means that increased offered load cause much more data frame has to transmit among nodes, so it give an opportunity to reuse time period like cases in the figure 3. The primary reason for the delay was because, if additional transmission was possible in the section fixing the exposed terminal problem (thus, if the section could not pick up the CTS frame and had a frame to send), it would transmit the frame after the timer was set, and additional frames would be transmitted for the lengthened transmission time due to the long delay in 
propagation. Results of the proposed protocol may seem to underperform, considering the end-to-end delay. However, in the figure 6, it is statistically superior, compared to the existing MACA protocol.

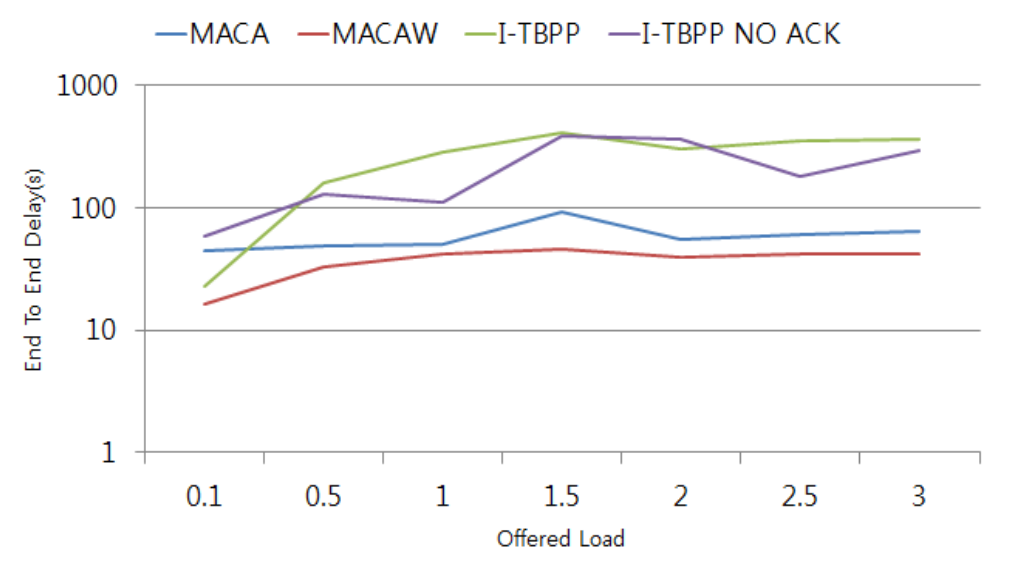

Figure 8. End-to-end Delay Comparison over Various Protocols

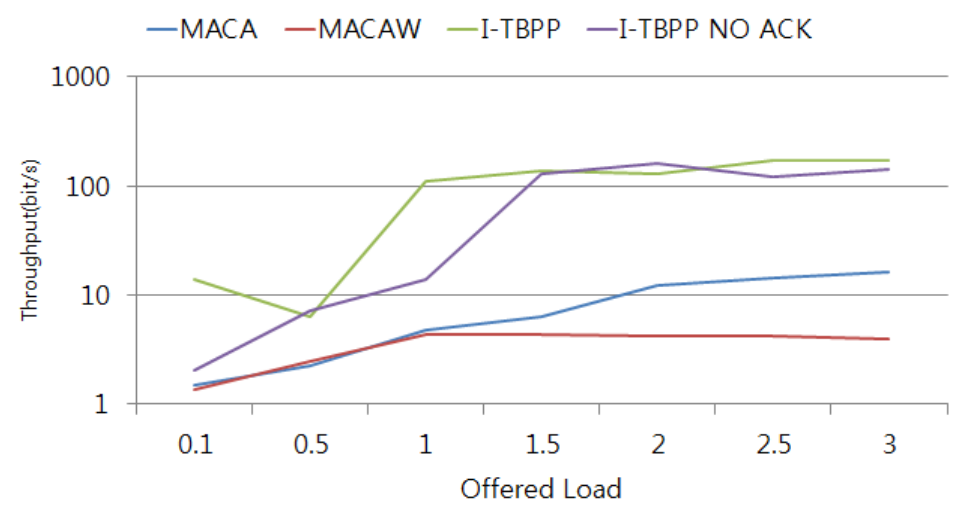

Figure 9. Throughput Comparison over Various Protocols

Figure 9 is the simulation results for throughput. Looking at each protocol's throughput results, the proposed protocol has higher throughput compared to MACA. This is because it reuses the space from the long propagation delay to preferentially send data to closer nodes, and solves the exposed terminal problem. Moreover, from just comparing I-TBPP with ACK and I-TBPP without ACK, the protocol using ACK had higher throughput, on average. After transmitting ACK, additional transmission would take place during the waiting time for this protocol, resulting in higher throughput.

\section{Conclusion}

Underwater wireless sensor networks are an emerging field to various applications like environment monitoring, habitat, and military surveillance and so on. Unfortunately, we could not use already developed protocols, like MAC protocol due to different transmit environment from terrestrial on the earth. In underwater, radio frequency has severe attenuation so we take acoustic sound wave to transmit data. Acoustic sound wave's characteristic is very different to radio in terms of propagation speed, bandwidth, attenuation model, and etc.

Considering that underwater packet transmission is extremely slow (compared to the ground-based wireless networks) due to long propagation delay, this research 
proposed a new MAC protocol. The proposed protocol preferentially processes closer nodes and solves the exposed terminal problem during a long propagation delay. As a result, the overall performance is better, compared to the existing wireless MAC protocol, MACA.

However, the proposed protocol has one drawback in that end-to-end delay is long. We think it is small fact and it give more throughput by the reuse of time period of long propagation delay wait time for almost waiting period during transmission phase. Further research will find solutions to shorten the end-to-end delay and apply them to the proposed protocol. For example, when we apply geographical information like position information of node will allow that use of geographical routing protocol and angle based decision when make decision for time overlapping. The proposed MAC protocol could get more precise decision with mixed information of distance and angle to neighbor node. In the future research, we will try to consider geographical information through the self-configuration of position information and try to get more precision decision algorithm for timing overlapping.

\section{References}

[1] L. S. Hun and C. C. Ha, "A Study on the Underwater Sensor Networks", The Korean Institute of Communications and Information Sciences, vol. 35, (2010), pp. 951-952.

[2] J. E. Kim, N. Y.Yun, Y. P. Kim, S. Y. Shin, S. H. Park, J. H. Jeon, S. J. Park, S. K. Kim and C. H. Kim, "Design and Performance Evaluation of Hierarchical Protocol for Underwater Acoustic Sensor Networks", The Korea Society for Simulation, vol. 20, (2007), pp. 157-166.

[3] X. Guo, M. R. Frater and M. J. Ryan, "A Propagation-delay-tolerant Collision Avoidance Protocol for Underwater Acoustic Sensor Networks”, In Proceedings of MTS/IEEE OCEANS, Boston, MA, USA, (2007).

[4] X. Guo, M. R. Frater and M. J. Ryan, "Design of a Propagation-delay-tolerant MAC protocol for Underwater Acoustic Sensor Networks", In Journal of OCEANIC ENGINEERING, vol. 34, (2009).

[5] H. H. Ng, W. S. Soh and M. Motani, "MACA-U: A Media Access Protocol for Underwater Acoustic Networks", In Conference of Global Telecommunications, (2008).

[6] N. Y. Yun, H. J. Cho and S. H. Park, "Neighbor nodes aware Mac Scheduling Scheme in Underwater Acoustic Sensor Network", In Conference of Computational Science and Engineering, vol. 2, (2009).

[7] L. Pu, Y. Luo, H. Mo, S. Le, Z. Peng, J. H. Cui and Z. Jiang, "Comparing underwater MAC protocols in real sea experiments", Computer Communications, vol. 56, (2015), pp. 47-59.

[8] N. Chirdchoo, W. S. Soh and K. C. Chua, "MACA-MN: A MACA-based MAC Protocol for Underwater Acoustic Networks with Packet Train for Multiple Neighbors", In Journal of OCEANIC ENGINEERING, (2008), pp. 46-50.

[9] T. Hu and Y. Fei, "DSH-MAC: Medium Access Control Based on Decoupled and Suppressed Handshaking for Long-delay Underwater Acoustic Sensor Network", Local Computer Networks (LCN), (2013), pp. 24-24.

[10] H. X. Tan, K. Winston and G. Seah, "Distributed CDMA-based MAC Protocol for Underwater Sensor Networks", Local Computer Networks (LCN), (2007), pp. 26-36.

[11] G. M. Lundy, M. Almquist and T.Oruk, "Specification, verification and simulation of a wireless LAN protocol: MACAW", Military Communications Conference, (1998).

[12] H. Rae and O. Hyun, "An Improved MAC Protocol for Underwater Acoustic Sensor Networks", Asiapacific Proceedings of Applied Science and Engineering for Better Human Life, vol. 10, (2016).

[13] P. Karn, "MACA-A new channel access method for packet radio", in ARRL/CRRL Amateur Radio 9th Computer Networking Conference, (1990).

\section{Authors}

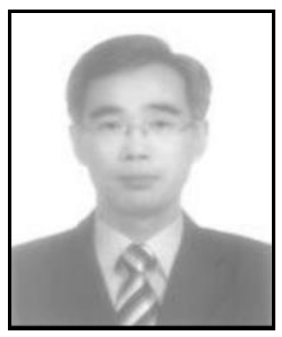

Seung-Hyun Oh, he received the B.S. degree in computer science from Dongguk University, Seoul, Korea, in 1988 and the M.S. and Ph.D. degrees in computer engineering from Dongguk University, Seoul, Korea, in 1998 and 2001, respectively. He has been a Professor with the Department of Computer Engineering, Dongguk University Gyeongju Campus since 2002. His current 
research interests include wireless communications and sensor networking system.

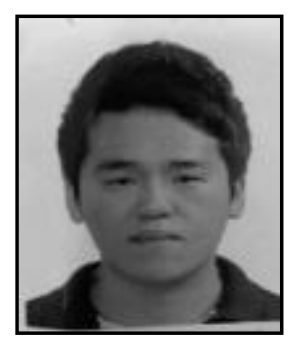

Bok-Rae Jang, he received the B.S. degree in computer engineering from Dongguk University, Gyeongju, Korea, in 2015 and he is currently in the Master course of Computer Science at Dongguk University. 